\title{
Immunosensor Characterization Using Impedance Spectroscopy
}

\author{
Lin $\mathrm{Ke}^{1 *}$, Wai Kwan Lim², New Siu Yee ${ }^{1}$, Xiao Di Su¹ and Bin Liu² \\ ${ }^{1}$ Institute of Materials Research and Engineering, Agency for Science, Technology and Research, 3 Research Link-117602, Singapore \\ ${ }^{2}$ Chemical and Biomolecular Engineering, NUS National University of Singapore-117 576, Singapore
}

\begin{abstract}
Non-invasive and high sensitive electrochemical impedance spectroscopy (EIS) has been used for qualitative characterization of stepwise surface preparation process for constructing an immunosensor, starting from plain silicon substrate, 3-aminopropyltriethoxysilane (APTES) silanization, glutaraldehyde functionalization and analytes (rat immunoglobulin G immobilization, followed by protein G-coated gold nanoparticles), using both the horizontal direction ( 2 terminal) and vertical direction (3 terminal) characterization setup. The investigation demonstrated that 2-terminal setup is able to review the quality of antibody and antigen molecules bonding and distinguish the effects from the underlayers on the immuosensors; while 3-terminal setup is able to correlate the thickness and dielectric constant of each layers. It correlated well to the incubation time of preparing different sensors as well. The two different EIS measurement setups are able to serve different needs for immunosensor characterization and EIS measurement shows higher sensitivities compared with UV-Vis absorption measurement.
\end{abstract}

Keywords: Impedance spectroscopy; Bio-receptor; Immunosensor

\section{Introduction}

In heterogeneous phase immunosensors, antibody and antigen interactions which exploited by covalent immobilization of antibody or antigen molecules on solid substrates are believed to be the most crucial step for the overall sensor performance [1]. In order to achieve the high sensitivity, high selectivity and prolonged device lifetime, the substrate materials must be modified to introduce the functional groups that can covalently bind the antibody or antigen with high bonding strength and stability $[2,3]$. Upon the chemical binding of both bio-receptor and analyte, the physiochemical property of the surface of sensor will change. Electrical impedance spectroscopy (EIS) system can be used to capture the impedance change on the sensor during the surface modification steps and analyte binding [4]. Renedo et al. [5] reviewed that by utilizing electrochemical data processing rather than optical method, immunosensor can function more sensitively to the subtle physiochemical change that they undergo during antibody-antigen interaction. For characterization of the Immunosensor, EIS system is used to capture the impedance change happened on the sensor during the analyte binding. An a.c. perturbation is introduced into the device, and it is expected that capacitance change will be significant as antibodyantigen binding is going to decrease the molecule conductivity and starting to accumulate dielectric charge instead [5,6]. Capacitance change in the assembled molecules (antibody-antigen) are recorded and analyzed when the sensor is functioning $[7,8]$.

In this research we aim to characterize an immunosensor in terms of its functionality and the quality during sensor assembly in electrolyte solutions at various stages of construction. even before and after analytical measurements to investigate the analyte change on the surface [9]. EIS is a powerful sensor characterization tool; it is non-invasive and does not disrupt device integrity [10]. As the sample immunosensor is designed to have multiple surface preparation layers, horizontal 2 terminal impedance responses will be used to analyze layers' quality and interface behavior. While vertical 3 terminal responses will be used to analyze the charge transfer, charge distribution in the overall devices and further correlate to the biosensor's functionality [11].

\section{Experiments and Instrumentations}

Plane Si substrate, substrate pretreatment and sensor construction

Silicon wafers of $150 \mathrm{~mm}$ diameter were cleaned with piranha solution to remove organic contaminants on the substrates. Cleaned and uncleaned Si substrates were both studied for comparison. The $\mathrm{Si}$ substrates (cleaned and uncleaned) were completely immersed into aminopropyltriethoxysilane (APTES) solution (5\% of APTES in ethanol). The substrates were left reacting in the solution for 2 hours at standard ambient temperature and pressure $\left(25^{\circ} \mathrm{C}\right.$ and approximately $1 \mathrm{~atm})$. Subsequently, the APTES-silanized Si substrates were soaked into glutaraldehyde (GA, $2.5 \mathrm{v} / \mathrm{v} \%$ in phosphate buffered saline, PBS) solution for 2 hours under standard ambient temperature and pressure.

$200 \mu \mathrm{L}$ of $50 \mu \mathrm{g} / \mathrm{mL}$ IgG solution (rate immunoglobulin G from Sigma Aldrich; diluted with PBS buffer) was pipetted onto Si substrates, and allowed to incubate on top of GA layer for 1 hour. The unoccupied surface was then blocked with $0.1 \%$ of bovine serum albumin (BSA). Next, $200 \mu \mathrm{L}$ of $20 \mathrm{nM}$ protein G-coated gold nanoparticles (i.e. pG-AuNPs) was incubated on top of Si substrates for 2 and 4 hours, respectively, before being characterized on EIS and UV-Vis spectroscopy. In between each association step, the Si substrates were rinsed with deionized water and blown dry to remove the unreacted and loosely bound samples.

\section{Preparation of gold nanoparticles and protein G-coated gold nanoparticles}

Figure 1 shows the schematic diagram of the preparation steps

*Corresponding author: $\mathrm{Ke} \mathrm{L}$, Institute of Materials Research and Engineering, Agency for Science, Technology and Research, 3 Research Link, Singapore-117602, Singapore, E-mail: karen-kl@imre.a-star.edu.sg

Received July 01, 2013; Accepted July 22, 2013; Published July 25, 2013

Citation: Ke L, Lim WK, New SY, Su XD, Liu B (2013) Immunosensor Characterization Using Impedance Spectroscopy. J Biosens Bioelectron 4: 138. doi:10.4172/2155-6210.1000138

Copyright: @ $2013 \mathrm{Ke} \mathrm{L}$, et al. This is an open-access article distributed under the terms of the Creative Commons Attribution License, which permits unrestricted use, distribution, and reproduction in any medium, provided the original author and source are credited. 


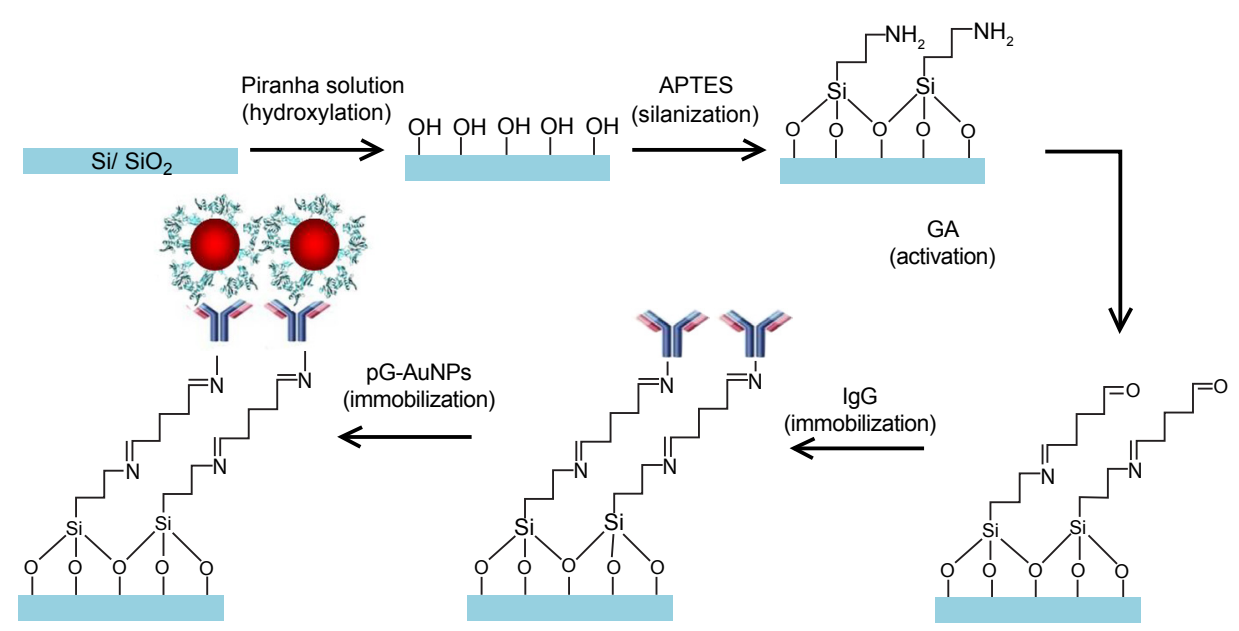

Figure 1: Scheme of the preparation steps for immunosensor study on Si substrate (PDB ID: 1FCC), which include piranha cleaning, silanization and immobilization steps.

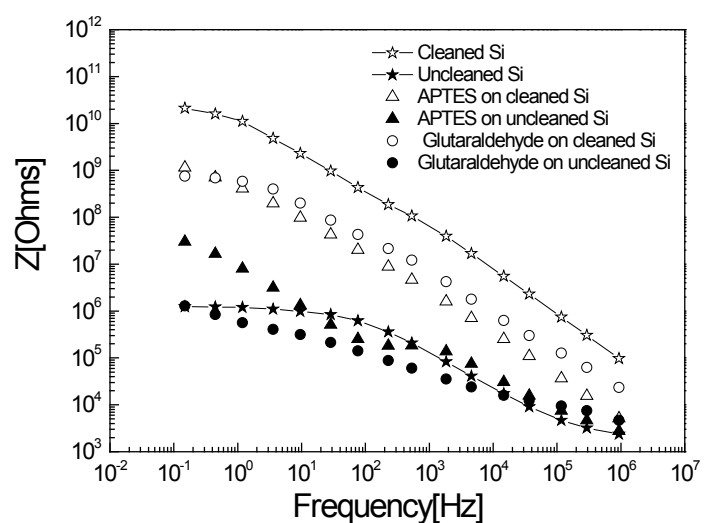

(a)

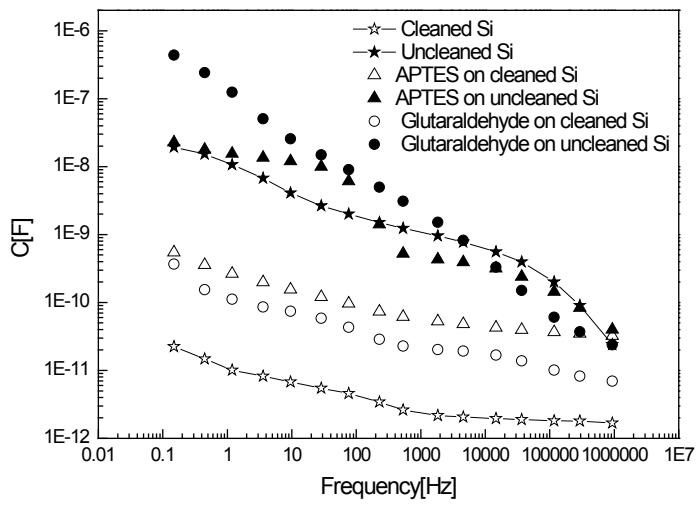

(b)

Figure 2: Impedance results versus frequency using 2 terminal test methode for clean and unclean Si substrates and APTES and Glutaraldehyude layers on top. $-{ }_{-}-$: Results on cleaned Si substrate, $\_$-: results on uncleaned Si substrate, $\triangle$ : Results on APTES surface on cleaned Si substrate, $\Delta$ : Results on APTES surface on uncleaned Si substrate, $\circ$ : Results on Glutaraldehyde surface on cleaned Si substrate, $\bullet$ : Results on Glutaraldehyde surface on uncleaned Si substrate. (a): Impedance $Z$ versus frequency $f$, (b) Capacitance $C$ versus frequency $f$.

for immunosensor study on Si substrate (PDB ID: 1FCC) [12]. $13 \mathrm{~nm}$ AuNPs were prepared according to literature procedure [13]. Briefly, $5 \mathrm{ml}$ of sodium citrate $(40 \mathrm{mM})$ was added to a boiling solution of $\mathrm{HAuCl}_{4}(50 \mathrm{~mL}, 1 \mathrm{mM})$. The solution was reflux for half an hour and then cold to room temperature while continue stirring. The molar concentration of the particles was calculated using Beer's Law: $A=\varepsilon b c$; with the calculated extinction coefficient, $\varepsilon=246661858 \mathrm{~L} \cdot \mathrm{cm}^{-1} \cdot \mathrm{mol}^{-1}$ (based on formula $\ln \varepsilon=k \ln D+a$; where $k=3.32111, a=10.80505$, [14], and D is the core diameter of NPs in $\mathrm{nm}$. To prepare protein G-coated gold nanoparticles (pG-AuNPs), $1 \mathrm{ml}$ of the as prepared bare AuNPs was added with $7 \mu \mathrm{l}$ of $1 \mathrm{mg} / \mathrm{ml}$ of protein G (Sigma Aldrich), and incubated for half an hour. $10 \mu \mathrm{l}$ of $1 \%$ PEG20k was then added to the solution before subjected for centrifugation at $14000 \mathrm{rpm}$. The pellet was resuspended in Mili-Q water and stored at $4^{\circ} \mathrm{C}$.

\section{Electrochemical Impedance Spectroscopy (EIS) and UV-VIS measurement}

The electrochemical impedance spectroscopy used in this research is INPHAZE High-Resolution EIS system ${ }^{\odot}$ by INPHAZE PTE LTD, with ultra high magnitude and phase resolution of $0.002 \%$ and $0.001^{\circ}$ respectively, over a frequency range of 1 milli-Hz to $1 \mathrm{Mega}-\mathrm{Hz}$, which is capable of accurate characterization of nano-level multilayer structures. The horizontal 2-terminal jig has been constructed to ensure uniform and consistently standard electrical circuit connection between the sample and the EIS system. The vertical 3-Terminal Chamber is one of the specialized sample chamber offered by INPHAZE EIS setup. The chamber is specially designed for characterization of devices in the domains of thin film, self-assembled monolayer, protein, lipid bilayer, and electrochemistry [2]. The 3-terminal chamber design focuses on consistent current flow distribution onto the sample; its electrolyte chamber on top of sample is reinforced with spring, in order to ensure unchanged positioning of measurement area and perfect sealing of chamber onto sample. The electrolyte used was $0.3 \mathrm{M}$ potassium chloride, diluted from $3 \mathrm{M}$-potassium chloride from Schott Instruments [2]. 4-wire resistance measurements are used to provide the most accurate way to measure small resistances by eliminating offset errors 
Citation: Ke L, Lim WK, New SY, Su XD, Liu B (2013) Immunosensor Characterization Using Impedance Spectroscopy. J Biosens Bioelectron 4: 138. doi:10.4172/2155-6210.1000138

associated with the test lead resistance in 2-wire ohms measurements. The low noise Au spring pins are used to contact with the samples to further improve the measurement accuracy.

Reflection UV-Vis spectroscopy (Shimadzu UV-3101PC) was used to characterize the test sample layers for comparative studies.

\section{Experimental Results}

\section{2-terminal characterization}

The blank Silicon substrate with and without Piranha cleaning are characterized using horizontal 2 terminal setup. Figures $2 \mathrm{a}$ and $2 \mathrm{~b}$ show the impedance $(\mathrm{Z})$ against frequency $(\mathrm{F})$ plots and capacitance (C) against frequency (F) plots, respectively. Substrates treated with APTES and glutaraldehyde on both the cleaned and uncleaned Silicon substrates are also tested, and the results are shown in Figures $2 \mathrm{a}$ and $2 \mathrm{~b}$, as well for comparison. APTES and glutaraldehyde treated substrates have lower overall impedance value than blank Silicon substrate using cleaned Si substrates. Both chemically treated substrates impedance profiles on cleaned $\mathrm{Si}$ and pure cleaned Silicon results are almost at synchronization for both $\mathrm{Z}$ and $\mathrm{C}$ plots. While from the impedance (Z) against frequency (F) plot and capacitance (C) against frequency (F) plot, both APTES and glutaraldehyde treated substrates using uncleaned Si substrates did not show any profile synchronization.

\section{3- terminal characterization}

The blank Silicon substrate with and without Piranha cleaning are characterized using 3 terminal setup. Figure $3 \mathrm{a}$ and $3 \mathrm{~b}$ shows the impedance $(\mathrm{Z})$ against frequency $(\mathrm{F})$ plot and capacitance $(\mathrm{C})$ against frequency (F) plot tested using 3 terminal setup. Substrates treated with APTES and glutaraldehyde on both the cleaned and uncleaned Silicon substrates are also tested, and the results are shown in Figures $3 \mathrm{a}$ and $3 \mathrm{~b}$, as well for comparison. Similarly, cleaned Silicon substrate

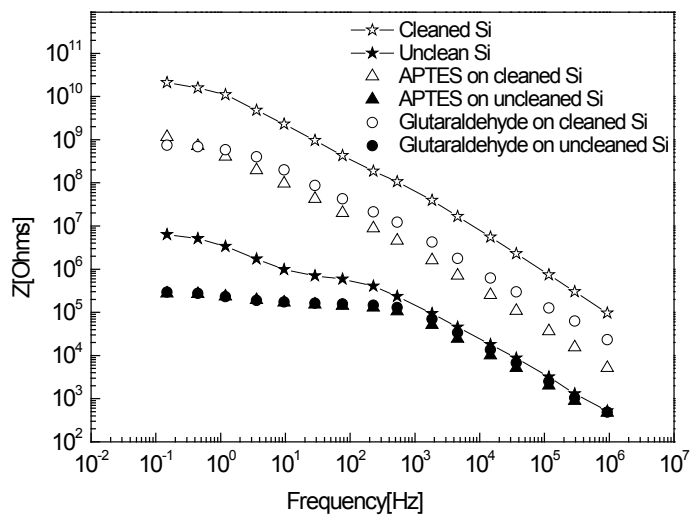

(a)

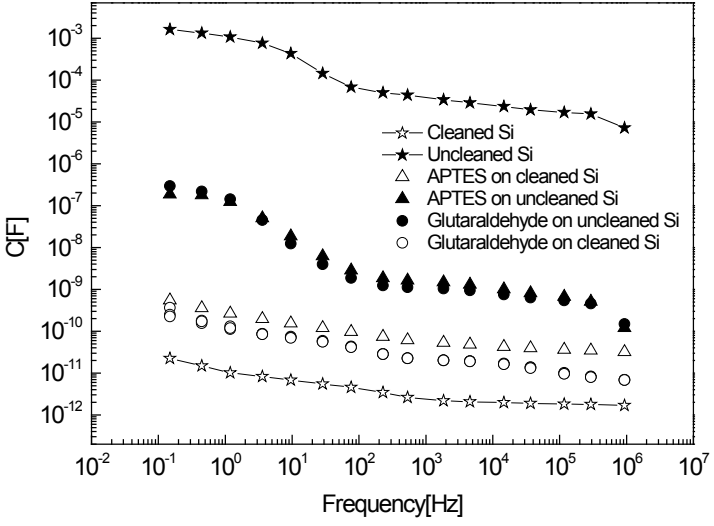

(b)

Figure 3: Impedance results versus frequency using 3 terminal test methode for clean and unclean Si substrates and APTES and Glutaraldehyude layers on top. - : Results on cleaned Si substrate, $\rightarrow$ : results on uncleaned Si substrate, $\triangle$ : Results on APTES surface on cleaned Si substrate, $\Delta$ : Results on APTES surface on uncleaned Si substrate, ॰: Results on Glutaraldehyde surface on cleaned Si substrate, $\bullet$ : Results on Glutaraldehyde surface on uncleaned Si substrate. (a): Impedance $Z$ versus frequency $f$, (b) Capacitance $C$ versus frequency $f$.

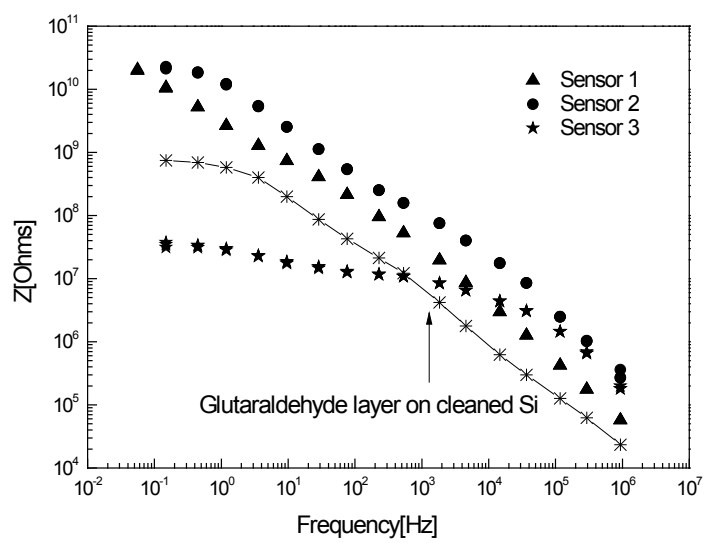

(a)

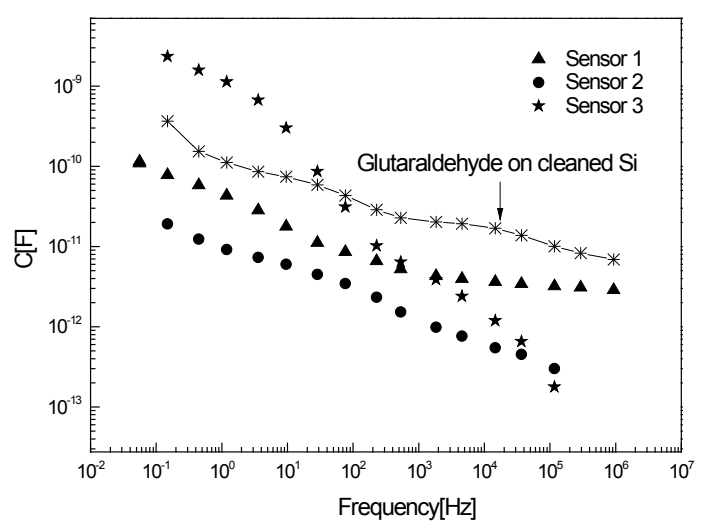

(b)

Figure 4: Comparative study of Sensors using 2-terminal characterization. $\mathbf{\Delta}$ : Sensor 1: Silicon based substrate with 2 hours of pG-AuNPs incubation period, $\bullet$ : Sensor 2: Silicon substrate with 4 hours of pG-AuNPs incubation period. $\star$ : Sensor 3: Silicon/Silicon Dioxide substrate with 4 hours of pGAuNPs incubation period. (a): Impedance $Z$ versus frequency $f$, (b) Capacitance $C$ versus frequency $f$. 
Citation: Ke L, Lim WK, New SY, Su XD, Liu B (2013) Immunosensor Characterization Using Impedance Spectroscopy. J Biosens Bioelectron 4: 138. doi:10.4172/2155-6210.1000138

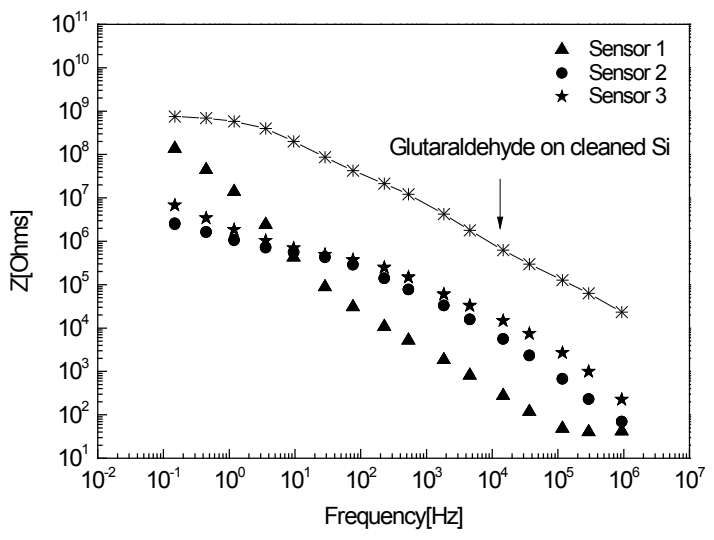

(a)

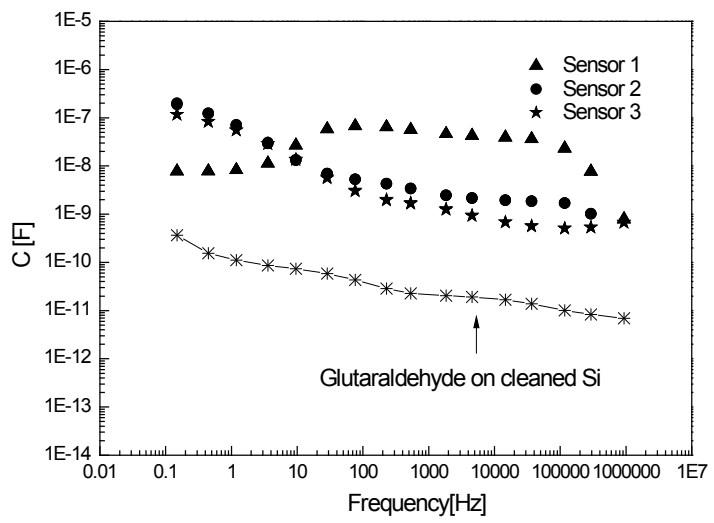

(b)

Figure 5: Comparative study of Sensors using 3-terminal characterization. $\mathbf{\Delta}$ : Sensor 1: Silicon based substrate with 2 hours of pG-AuNPs incubation period, •: Sensor 2: Silicon substrate with 4 hours of pG-AuNPs incubation period. $\star$ : Sensor 3: Silicon/Silicon Dioxide substrate with 4 hours of pGAuNPs incubation period. Result on Glutaraldehyde surface on cleaned Si substrate is also shown for comparison. (a): Impedance Z versus frequency $\mathrm{f}$, (b) Capacitance $\mathrm{C}$ versus frequency $\mathrm{f}$.

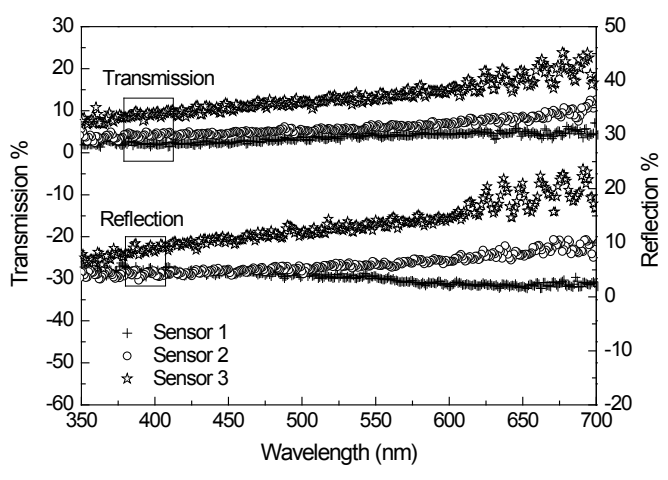

(a)

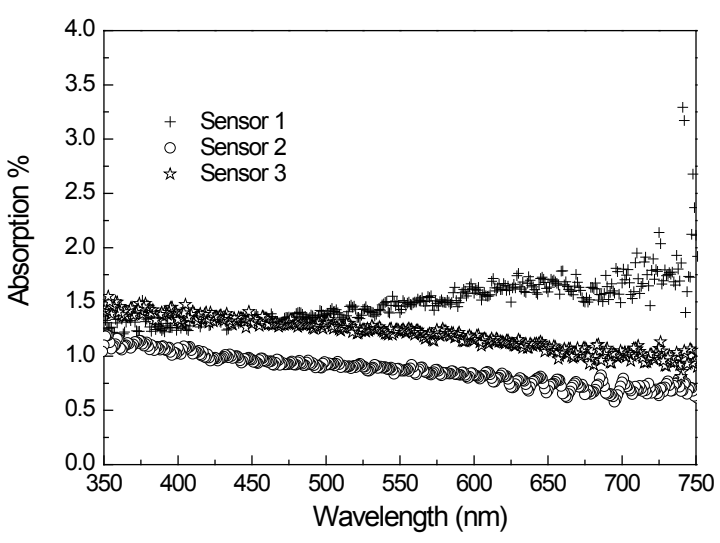

(b)

Figure 6: Comparative study of the sensors' absorptivity, transmissivity, and reflectivity. +: Sensor 1: Silicon based substrate with 2 hours of pG-AuNPs incubation period, $\circ$ : Sensor 2: Silicon substrate with 4 hours of pG-AuNPs incubation period. hours of pG-AuNPs incubation period. Results on Glutaraldehyde surface on cleaned Si substrate is also shown for comparison. (a): Transmission and reflection curves, (b) Absorption curves.

shows obvious higher impedance than the uncleaned Silicon substrate. Lower capacitance for the cleaned $\mathrm{Si}$ than the uncleaned $\mathrm{Si}$ is also demonstrated. Like the result from 2-terminal characterization of blank-APTES-glutaraldehyde Silicon substrate, 3-terminal test result also displays that APTES and glutaraldehyde treated substrates have lower overall impedance value than blank Silicon Si substrates, and capacitance of APTES-glutaraldehyde substrates is also generally higher than blank Si. Both chemically treated substrates impedance profiles and cleaned Silicon results are almost at synch in all 3 of the analytical graphs in both $\mathrm{Z}$ and $\mathrm{C}$ plots.

\section{Sensor setup and characterization}

3 types of sensors have been constructed and compared. Each substrate was functionalized with IgG (bio-receptor molecule), followed by $\mathrm{pG}$-AuNPs with different incubation time. The nomenclature of these biosensors is: Sensor 1: Silicon based substrate with 2 hours long of pG-AuNPs incubation period. Sensor 2: Silicon substrate with 4 hours long of pG-AuNPs incubation period. Sensor 3: Silicon/Silicon Dioxide substrate with 4 hours long of pG-AuNPs incubation period.

Figures $4 \mathrm{a}$ and $4 \mathrm{~b}$ show the impedance and capacitance profiles for Sensor 1 to Sensor 3 measured with 2-terminal setup. The curves with glutaraldehyde as top layer on cleaned Si surface using 2-terminal setup are also shown. Sensor 1 and Sensor 2 show very good congruent curves both in $\mathrm{Z}$ and $\mathrm{C}$ curves with the Glutaraldehyde layer except in the capacitance curve, the 2 sensors with different pG-AuNPs incubation time have deviations occurred at high frequency, and a slightly deviations occurred at lower frequency in the impedance curve. Sensor 3 is fabricated from different Silicon base substrate, which is predicated to have diffident impedance and capacitance trends. Figures $5 \mathrm{a}$ and $5 \mathrm{~b}$ show the impedance and capacitance profile for Sensor 1 to Sensor 3 measured using 3-terminal setup. The curves done on the glutaraldehyde layers on cleaned Si surface using 3-terminal setup are also shown. Deviation from Sensor 1 using recommended 2 hours 
incubation period for immobilize AuNPs-IgG recommended from literature is significant, as both Sensor 2 and Sensor 3 underwent longer period of analyte incubation of 4 hours. The capacitance versus frequency figure showed the consistent results, Sensor 2 and Sensor 3 demonstrated the congruent curves with the glutaraldehyde surface results, but higher capacitance founded in Sensor 1 in middle and high frequency ranges.

\section{UV-VIS spectroscopy study}

The Ultraviolet-Visible Spectroscopy is used to characterize the biosensors devices in the visible light region from 300-750 nm. Figure 6 left shows the transmissivity and reflectivity study of sensors 1, 2 and 3 in the visible region. All the measurements above were made by using blank Silicon substrate as reference. With the prolonged incubation period of pG-AuNPs, the transmissivity and reflectivity increase. The transmissivity and reflectivity of both long incubation period sensors (Sensor 2 and Sensor 3) increases. Sensor 3 with the $\mathrm{SiO}_{2}$ substrate possesses even higher transmissivity and reflectivity than Sensor 2. Figure 6 right shows the absorption profiles of all 3 sensors, overall the absorption are low as the layers are thin and pG-AuNPs particle concentrations are low. There is no noticeable absorbance at $550 \mathrm{~nm}$ region. Sensor 1 shows the highest absorption over the range of $350 \mathrm{~nm}$ to $750 \mathrm{~nm}$, followed by Sensor 3 and Sensor 2 .

\section{Analyses and Discussion}

EIS is very sensitive to detect surface change; cleaned Silicon substrate shows obviously higher impedance than the uncleaned Silicon substrate. In the low frequency region, impedance difference between uncleaned and cleaned substrates is more significant than the medium and higher frequency region. The organic contaminants/greases adsorbed onto the Silicon surface cause the Si surface lesser resistive, and the effects contributed largely to the lower frequency range. Lower capacitance for the cleaned $\mathrm{Si}$ than the uncleaned $\mathrm{Si}$ is demonstrated. This is most likely caused by the heightened double layer dielectric properties, as both $\mathrm{Si}$ and membrane of fatty acid molecule (grease/ contaminant) have large charge storage capacity. [2] Grease molecules have carboxylate end $(-\mathrm{COOH})$ as functional group and APTES has an amine (-NH $\mathrm{NH}_{2}$ functional group. Both functional groups can be bonded together through simple neutralization reaction or formation of amide. As they are bonded together, this relatively large organic molecule could have a high dielectricity than just silanized APTES on cleaned substrate. APTES and glutaraldehyde treated substrates have lower overall impedance value than blank Silicon substrate using cleaned Si substrates. Both chemically treated substrates impedance profiles and cleaned Silicon results are almost at synch in all 3 of the analytical graphs in both Z and C plots [15]. The similarity in both APTES and glutaraldehyde substrate profiles is explained on the molecular binding approach. As APTES molecules started to form covalent silane bond with $\mathrm{Si}$ on the surface, it takes on a modified impedance profile from the blank Si profile $[16,17]$. Glutaraldehyde molecules are next bonded at the end of APTES chain, thus the glutaraldehyde-treated substrate would be congruent with APTES-treated substrate profile. These congruous profiles from the experimental result proved the uniformity of pre treatment chemicals on the Si surface. It was further proved that the impedance $(\mathrm{Z})$ against frequency $(\mathrm{F})$ plot and capacitance (C) against frequency (F) plot, for both APTES and glutaraldehyde treated substrates using uncleaned $\mathrm{Si}$ substrates, did not show any profile synchronization. In summary, as the impedance profiles between APTES and glutaraldehyde on cleaned Si substrate are highly congruent, it is safe to assume that both layers share the same molecular orderly arrangement. The substrate and surface quality will affect the subsequent above layers' quality. The impedance profile can be used to determine the wellness of molecular adhesion above the substrate sequentially.

Similarly, cleaned Silicon substrate shows obvious higher impedance than the uncleaned Silicon substrate. Lower capacitance for the cleaned Si than the uncleaned $\mathrm{Si}$ is also demonstrated. Like the result from 2-terminal characterization of blank-APTES-glutaraldehyde Silicon substrate, 3-terminal test result also displays that APTES and glutaraldehyde treated substrates have lower overall impedance value than cleaned blank Silicon Si substrates, and capacitance of APTESglutaraldehyde substrates is also generally higher than blank Si. Both chemically treated substrates impedance profiles and cleaned Silicon results are almost at synch in all 3 of the analytical graphs in both $\mathrm{Z}$ and $\mathrm{C}$ plots.

Both 2-terminal and 3-terminal characterization adopt explicitly different mechanism to measure the sample device. For the horizontal 2-terminal configuration, electrical a.c. flows though the immobilized molecules along the surface, the under layers and interfaces quality will determine the overall impedance performance measured by 2-terminal setup. On the other hand, 3-terminal directs the a.c. in the perpendicular direction from substrates to the surface. Each layers and interfaces quality will also demonstrate the effects on the overall impedance response curves. Therefore, both 2-terminal and 3-terminal measurement setups can reveal the layers and interfaces quality as shown in the similar measurement results and trends using both setups. Furthermore 3-terminal setup could bring us more information with higher amplitude and phase resolution impedance measurements. Each layers, e.g. APTES, glutaraldehyde and interfaces RC constants can be correspondingly derived and monitored. This could be significantly useful in monitoring each individual layer/interfaces' effects on the overall sensor performance [18].

Based on the above results of the multiple layers constructed on $\mathrm{Si}$ substrates, the mechanism behind the Sensors' performance is revealed. Sensor 1 and Sensor 2 show very good congruent curves both in $\mathrm{Z}$ and $\mathrm{C}$ curves, with the Glutaraldehyde layer except in the capacitance curve, the 2 sensors with different pG-AuNPs incubation time have deviations occurred at high frequency and a slightly deviations occurred at lower frequency in the impedance curve. Such congruence in data profile using 2-terminal setup is due to both Sensor 1 and Sensor 2 is fabricated from the same Silicon substrate. Sensor 3 is fabricated from different Silicon base substrate, which is predicated to have diffident impedance and capacitance trends. Same conclusions can be drawn that the 2-terminal EIS setup has the capabilities to discern the device base layers. The under layers quality will affect the upper layers' performance magnificently. With gold nanoparticles (AuNPs) treated IgG formed on the surface, both Sensor 1 and Sensor 2 shows increased impedance value compared with glutaraldehyde layer results. With longer incubation time, the AuNPs- IgG layer formed for Sensor 2 will be thicker than Sensor 1; Sensor 2 shows even higher impedance value. With the slightly increased AuNPs concentration in Sensor 2 as well, low frequency impedance plot demonstrated a slightly decrease. With AuNPs-IgG formed on the surface, both Sensor 1 and Sensor 2 show decreased capacitance value. The obvious high frequency capacitance deviation among Sensor 1, Sensor 2 and Glutaraldehyde layer results was due to the concentration of AuNPs are different, which determined the different responses of the sensors in the high frequency range; the higher the AuNPs concentration, the lower the capacitance will be detected. The frequency turning points in the capacitance versus 
frequency plot can be used to correlate the AuNPs concentration [1921]. Both Sensor 2 and Sensor 3 have congruous impedance profile with the curve done on the surface of glutaraldehyde, while the impedance is much lower. The reasons could be due to the AuNPs improved the vertical resistance in the very thin layer, while they did not show much effects on the horizontal resistance with longer measurement distance. Deviation from Sensor 1 using recommended 2 hours incubation period for immobilize AuNPs-IgG recommended from literature is significant, as both Sensor 2 and Sensor 3 underwent longer period of analyte incubation of 4 hours. With nanoparticle attached Au-IgG, it might need more time to completely form uniform antigen-antibody complex. The extra time needed for complex formation with antigencoated AuNPs is proposed to be caused by unforeseen interruption of nanoparticle onto the binding kinetics of the conventional antibodyantigen complex. The capacitance versus frequency figure showed the consistent results, Sensor 2 and Sensor 3 demonstrated the congruent curves with the glutaraldehyde surface results, but higher capacitance founded in Sensor 1 in middle and high frequency ranges. Sensor 1 with shorter incubation time than Sensor 2 and Sensor 3 showed the different behaviour in different frequency range, the reasons could be due to the incomplete formation of antigen-antibody complex. The 3-terminal characterization is capable of deliberately showing the difference of analyte incubation period from impedance and capacitance data profile. The substrate difference, Si with and without $\mathrm{SiO}_{2}$ which slightly modified the substrate surfaces, showed little effect on the impedance and capacitance response in the vertical direction measurement.

The Ultraviolet-Visible Spectroscopy is used to characterize the biosensors devices in the visible light region from 300-750 nm. With the prolonged incubation period of pG-AuNPs, the transmissivity and reflectivity increase. The transmissivity and reflectivity of both long incubation period sensors (Sensor 2 and Sensor 3) increases. With the increased incubation time, the IgG and pG-AuNPs complexes are formed more uniformly. Therefore, the reflection and transmission enhanced. Sensor 3 with the $\mathrm{SiO}_{2}$ substrate possesses even higher transmissivity and reflectivity than Sensor $2 . \mathrm{SiO}_{2}$ acted as an optical spacer between the substrate and the following biosensor layers which resulted in a redistribution of the optical electric field [22]. The effects of the $\mathrm{SiO}_{2}$ interface can be manifested not only in optical characterization, but also in electrical characterization, as presented in the impedance results previously with increased impedance and decreased capacitance compared to Sensor 2. For the absorption curves, there is no obvious $550 \mathrm{~nm}$ AuNPs peaks presented as the concentration of the AuNPs is relatively small, however, in the impedance spectroscopy curves, there are obvious deviations for all three sensors from the curves with glutaraldehyde as top layer on cleaned Si surface as shown in (Figures 4 and 5). This shows that the high phase and amplitude resolution impedance spectroscopy demonstrated higher sensitivity compared with optical UV-Vis method and potential usage for in-situ biosensor's fabrication and quality monitoring.

\section{Conclusions}

Cleaned and contaminated silicon substrates were investigated using EIS in this study. EIS detects a surge in substrate sample impedance caused by the insulating fatty acids from the contaminant grease. APTES and glutaraldehyde layers are deposited on the substrates sequentially and their molecule arrangement was investigated. It can be concluded that 2-terminal horizontal setup can be used as a measure of how well the molecules are arranged as the topography of substrate surface is directly molding the topographic of the upper layers, while
3 terminal vertical setups can be used to extract each layers and interfaces' information. Experimental results on biosensors showed clear correlation of the $\mathrm{pG}$-AuNPs incubation time with the impedance response. In summary, our demonstrated vertical and horizontal experimental results on substrate with different layer treatment and biosensor construction is useful, because by alternating the configuration of EIS setup, one may discover some undetected salient feature of substrates and bottom layers effects on the subsequent top layers and biosensors performances, which is more informative than the optical UV-Vis spectroscopy characterization methodology.

\section{References}

1. Tuan VD (2003) Biomedical photonics handbook. CRC Press, USA.

2. Wang $\mathrm{ZH}$, Jin $\mathrm{G}$ (2004) Covalent immobilization of proteins for the biosensor based on imaging ellipsometry. J Immunol Methods 285: 237-243.

3. Zhao W, Xu JJ, Chen HY (2006) Electrochemical Biosensors based on layerby-layer assemblies. Electroanalysis 18: 1737-1748.

4. Daniels JS, Pourmand N (2007) Lable-free impedance biosensors: Opportunities and challenges. Electroanalysis 19: 1239-1257.

5. Renedo OD, Alonso-Lomill MA, Martinex MJA (2007) Recent developments in field of screen-printed electrodesand their related applications. Talanta 73 202-219.

6. Brett C (2008) Electrochemical impedance spectroscopy for characterization of electrochemical sensors and biosensors. ECS Trans 13: 67-80.

7. Grieshaber D, Mackenzie R, Voros J, Reimhult E (2008) Electrochemical biosensors-sensor principles and architectures. Sensors 8: 1400-1458.

8. Rogers KR (2000) Principles of affinity-based biosensors. Molr Biotechnol 14 109-129.

9. Macdonald JR (1991) Impedance Spectroscopy. Ann Biomed Eng 20: 289-305

10. Pohanka M, Skladal P (2008) Electrochemical biosensors-principles and applications. J Appl Biomed 6: 57-64.

11. www.inphaze.com.au

12. Sauer-Eriksson AE, Kleywegt GJ, Uhlen M, Jones TA (1995) Crystal structure of the $\mathrm{C} 2$ fragment of Streptococcal protein $\mathrm{G}$ in complex with the $\mathrm{Fc}$ domain of human IgG. Structure 3: 265-278

13. Tan YN, Su X, Liu ET, Thomsen JS (2010) Gold-nanoparticle-based assay for instantaneous detection of nuclear hormone receptor-response elements interactions. Anal Chem 82: 2759-2765.

14. Liu X, Atwater M, Wang J, Huo Q (2007) Extinction coefficient of gold nanoparticles with different sizes and different capping ligands. Colloids Surf B Biointerfaces 58: 3-7.

15. Heinz H, Vaia RA, Farmer BL (2008) Relation between packing density and thermal transitions of alkyl chains on layered silicate and metal surfaces. Langmuir 24: 3727-3733.

16. Tao $F(2008)$ Nanoscale surface chemistry in self- and directed-assembly of organic molecules on solid surfaces and synthesis of nanostructured organic architectures. Pure Appl Chem 80: 45-57.

17. Inphaze Pty Ltd. () User Manual-How to Use the Inphaze 3-Terminal Chamber.

18. Lasia A (2002) Electrochemical impedance spectroscopy and its applications. Modern Aspects of Electrochemistry 32: 143-248.

19. Lillard, RS, Moran PJ, Isaacs HS (1992) A novel method for generating quantitative local electrochemical impedance spectroscopy. J Electrochem Soc 139: 1007-1012.

20. Jain PK, Lee KS, El-Sayed IH, El-Sayed MA (2006) Calculated absorption and scattering properties of gold nanoparticles of different size, shape, and composition: Applications in the biological imaging and biomedicine. J Phys Chem 110: 7238-7248

21. Chen FC, Wu JL, Hung Y (2010) Spatial redistribution of the optical field intensity in inverted polymer solar cells. Appl Phy Lett 96: 193304-193306.

22. Pucker G, Bellutti P, Cazzanelli M, Gaburro Z, Pavesi L (2001) ( $\left.\mathrm{Si} / \mathrm{SiO}_{2}\right)_{n}$ multilayers and microcavities for LED applications. Opt Mat 17: 27-30. 